\title{
Super-Resolution Definition of Coordinates of Single Semiconductor Nanocrystal (Quantum Dot): Luminescence Intensity Dependence
}

\author{
M.Yu. Eremchev ${ }^{1,2,3 a}$, I.Yu. Eremchev ${ }^{2}$, and A.V. Naumov ${ }^{2,4}$ \\ ${ }^{1}$ Moscow Institute of Physics and Technology, 9 Institutskiy per., Dolgoprudny, Mocsow Region, 141700, Russia \\ ${ }^{2}$ Institute for Spectroscopy RAS, 5 Fizicheskaya str., Troitsk, Moscow, 142190, Russia \\ ${ }^{3}$ Skolkovo Institute of Science and Technology, 3 Nobelya str., Moscow, 143026, Russia \\ ${ }^{4}$ Moscow State Pedagogical University, 29 M.Pirogovskaya str, Moscow 119991, Russia
}

\begin{abstract}
In this research a relation between the accuracy of restoration of the single quantum dots (QD) $\mathrm{CdSe} / \mathrm{CdS} / \mathrm{ZnS}$ cross-cut coordinates and luminescence intensity was investigated. It was shown that the limit of the accuracy of determining the coordinates of a single QD for a considerable total amount of registered photons approaches its limiting value that is comparable to the size of the QD. It also means that the installation used in the research is mechanically stable enough to reach the limiting values of determination accuracy of point emitters coordinates.
\end{abstract}

Keywords: quantum dots, single photon sources, super-resolution fluorescence microscopy

One of the potentially topical applications of employment luminescent quantum dots is using these as nanomarkers in the fluorescence super-resolution microscopy and related areas. [1-3] As far as QD sizes are by far less than the registered radiation wavelength, QD coordinates could be restored (by the same way as for single organic molecules [4]) with the accuracy dramatically exceeding the diffraction limit. In this connection important point is creating methods of restoring cross-cut coordinates of single QD and investigation influences of different conditions on the accuracy of determining the QD coordinates. Determining factor in this case is the total amount of effective photons registered during visualization of single QD.

In this research the visualization of single QDs was conducted by registering luminescence from a single QD using the wide-field epi-luminescence microscope and cooled CCD camera with an internal electronic multiplication (detailed description of installation is given in [5]) and excited by the continuous-wave laser. To get the information about the QD luminescence intensity and the QD coordinates computing of CCD camera frames were done using approximation of QD images (Airy disk) by the symmetrical two-dimensional Gaussian functions. Collected peak positions values of Gaussian correspond to the QD locations, and the integral of Gaussian function - to luminescence intensity.

In the measurements we used core-shell nanocrystals $\mathrm{CdSe} / \mathrm{CdS} / \mathrm{ZnS}$ with $\mathrm{Si}$-organic ligands (manufactured by QD-Light, Russia). The QDs were strongly diluted in toluene and deposited on the glass substrate by spin-coating.

${ }^{\text {a } C o r r e s p o n d i n g ~ a u t h o r: ~ e r e m c h e v . m y @ p h y s t e c h . e d u ~}$

This is an Open Access article distributed under the terms of the Creative Commons Attribution License 4.0, which permits unrestricted use, distribution, and reproduction in any medium, provided the original work is properly cited. 
Sequential detection of a big amount of QD luminescence images allows to determine QD coordinates with high statistical confidence as well as statistical dispersion for all images and thus to get average value and accuracy (standard deviation) of defining QD coordinates for each storage time. (Fig. 1). In the set of 10000 sequential measurements (10000 frames) with $10 \mathrm{~ms}$ storage time dispersion value was approximately $10 \mathrm{~nm}$ but actual QD (CdSe) size is $\sim 5.2 \mathrm{~nm}$. For the same set of experiments and two times greater storage time standard deviation was $8 \mathrm{~nm}$. Thus, increasing in the storage time caused increase in the restoration accuracy of QD coordinates. This result is intuitively clear - increase in storage time equals to increase in total amount of photons detected from a single QD in a set.

QD luminescence track represents ever-changing in time intensity curve - alternation of on-/offand grey states (so-called blinking [6,7]). That is why further elaboration was conducted for "isointensive" levels corresponding to the different intensity values. The width of these levels is chosen sufficiently small but at the same time each sampling included enough points for good statistical confidence (about 350 points). For each intensity level average value and dispersion of coordinates were determined and using this data dependence between dispersion value and intensity was plotted. This dependence corresponds closely to semi-empirical formula [8].

a)

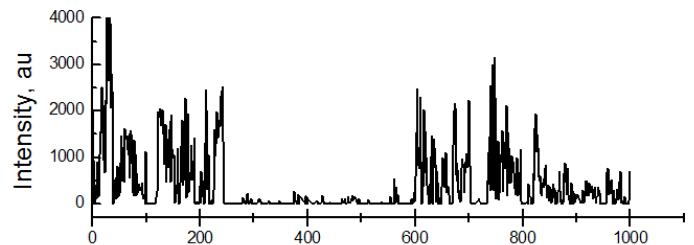

b)

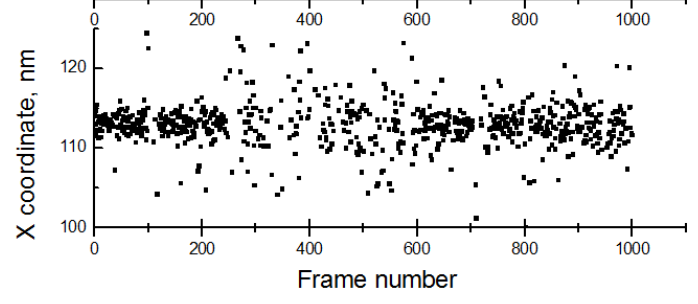

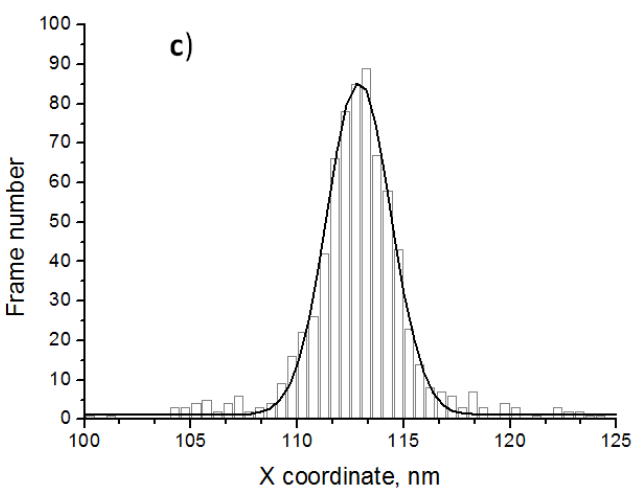

Figure 1. Fluorescence trace (a) and corresponding reconstructed $\{X\}$ spatial coordinate (b) of single core-shell quantum dot $\mathrm{CdSe} / \mathrm{CdS} / \mathrm{ZnS}$ measured with exposure bin time of $100 \mathrm{~ms}$ under excitation by $\mathrm{cw}$-laser (532 nm). The histogram (c) demonstrate the dispersion of reconstructed $\{\mathrm{X}\}$ coordinate for the selected QD as measured in different video CCD-frames for the time moments with brightest QD luminescence. A few nm accuracy of lateral coordinate reconstruction is achievable.

This research shows that the limit of the accuracy of determining the coordinates of a single QD for a considerable total amount of registered photons approaches its limiting value that is comparable to the QD size. It appears from this that the installation used in the research is mechanically stable enough to reach the limiting values (few nanometers) of determination accuracy of the QD coordinates.

The support from Russian Foundation for Basic Researches is acknowledged (14-02-00822).

\section{References}

1. S. Weisenburger, V. Sandoghdar, Contemp. Phys. 56, 123 (2015)

2. J.Q. Xu, K.F. Tehrani, P. Kner, ACS NANO 9, 2917 (2015)

3. K.R. Karimullin, A.V. Naumov, J. Lumin. 152, P.15 (2014)

4. A.V. Naumov, Physics Uspekhi. 56, P.605 (2014)

5. A.V. Naumov, I.Yu. Eremchev, A.A.Gorshelev, Eur. Phys. J. D. 68, 348 (2014)

6. K.T. Shimizu, R.G. Neuhauser, et. al., Phys. Rev. B 63, 205316 (2001)

7. A. L. Shcukina, I. Yu. Eremchev, A. V. Naumov, "Looking at a blinking quantum emitter through time slots: The effect of blind times" Phys. Rev. E. Article ID : LE15452E (2015)

8. K.I. Mortensen, L.S. Churchman, J.A. Spudich, H. Flyvbjerg, Nat. Methods. 7, 377 (2010) 